\title{
SCENARIOS FOR BEAM COMMISSIONING OF THE LHC COLLIMATION SYSTEM
}

\author{
C. Bracco ${ }^{1)}$ R. W. Assmann, S. Redaelli, G. Robert-Demolaize \\ CERN, Geneva, Switzerland
}

\begin{abstract}
A system of collimators has been designed to protect the superconducting LHC magnets against quench and damage from the high intensity proton beams. The considerable number of collimators and the resulting number of degrees of freedom for their set-up require a well prepared commissioning strategy. Efficiency studies for various implementations of the LHC collimation system have been performed, taking into account the evolution in optics and beam intensity according to the LHC commissioning schedule. This paper explains the present plans for the setup sequence of collimators.
\end{abstract}

${ }^{1)} \mathrm{PhD}$ student, Ecole Polytechnique Fédérale de Lausanne (EPFL)

CERN, 


\title{
Scenarios for Beam Commissioning of the LHC collimation system
}

\author{
C. Bracco*, R.W.Assmann, S.Redaelli, G.Robert-Demolaize, CERN, Geneva, Switzerland
}

\section{Abstract}

A system of collimators has been designed to protect the superconducting LHC magnets against quench and damage from the high intensity proton beams. The considerable number of collimators and the resulting number of degrees of freedom for their set-up require a well prepared commissioning strategy. Efficiency studies for various implementations of the LHC collimation system have been performed, taking into account the evolution in optics and beam intensity according to the LHC commissioning schedule. This paper explains the present plans for the setup sequence of collimators.

\section{INTRODUCTION}

In the large hadron collider (LHC) two $7 \mathrm{TeV}$ proton beams will circulate storing an energy of $362 \mathrm{MJ}$ each. The quench limit of the LHC superconducting (sc) magnets is around $5 \mathrm{~mW} / \mathrm{cm}^{3}$ [1]. In order to avoid the quench of these magnets, a powerful system of collimators has been designed to absorb beam losses in dedicated warm regions of the machine.

Primary (TCP) and secondary (TCSG) collimators plus absorbers (TCLA) build up the two main cleaning insertions of the LHC ring: momentum (IR3) and betatron (IR7) cleaning. Diluter elements (TCDQ) and a TCSG protect the machine against mis-kicked beams in the dumping region (IR6). In Table 1 the nominal halfgaps of these collimators at $450 \mathrm{GeV}$ and $7 \mathrm{TeV}$ are presented in units of $\sigma=\sqrt{\varepsilon \beta}$ ( $\varepsilon$ is the beam emittance and $\beta$ is the beta-function). Typical beam size values are: $1 \sigma(450 \mathrm{GeV}) \sim 1 \mathrm{~mm}, 1 \sigma(7 \mathrm{TeV}) \sim 0.2 \mathrm{~mm}$.

Table 1: Nominal collimator half-gaps @ $450 \mathrm{GeV}$ and $7 \mathrm{TeV}$ in $\sigma$ units

\begin{tabular}{|l|ccc|ccc|}
\hline & \multicolumn{3}{|c|}{ 450GeV } & \multicolumn{3}{c|}{ 7 TeV } \\
\hline & TCP & TCSG & TCLA & TCP & TCSG & TCLA \\
IR3 & 8.0 & 9.3 & 10.0 & 15.0 & 18.0 & 20.0 \\
IR7 & 5.7 & 6.7 & 10.0 & 6.0 & 7.0 & 10.0 \\
\hline & TCSG & TCDQ & TCSG & TCDQ \\
IR6 & 7 & 8 & \multicolumn{3}{c|}{7.5} & 8 \\
\hline & TCLI & TDI & \multicolumn{3}{c|}{ TCT } \\
IR2/8 & 6.8 & 6.8 & \multicolumn{3}{c|}{8.3} \\
\hline & \multicolumn{1}{|c|}{} & & TCT & TCLP \\
IR1/5 & & & & 8.3 & 10 \\
\hline
\end{tabular}

During injection these settings must be implemented with the injection protectors (TDI, TCLI) set at $6.8 \sigma$.

\footnotetext{
* PhD student, École Polytechnique Fédérale de Lausanne (EPFL).
}

Tertiary collimators (TCT, half-gap $=8.3 \sigma$ for nominal $\beta^{*}=0.55 \mathrm{~m}$ ) are placed up-stream of the interaction points (IP) to protect the triplets. Special absorbers (TCLP set at $10 \sigma$ ) down-stream of IP1 and IP5 must catch the physics debris coming out from the high luminosity experiments during collision.

The performance of the collimation system is characterized by the global cleaning inefficiency $\eta$. This parameter represents the number of particles lost $\left(\mathrm{N}_{\text {lost }}\right)$ on the cold aperture of the machine, divided by the number of particles absorbed by the collimators $\left(\mathrm{N}_{a b s}\right)$. Through dedicated tracking simulations (SixTrack) and a detailed aperture model of the accelerator, the local cleaning inefficiency $\eta_{c}\left[\mathrm{~m}^{-1}\right]$ can be computed over small sections with length $\Delta \mathrm{s}$ (typically $0.1 \mathrm{~m}$ ) [2]:

$$
\eta_{c}=\frac{N_{\text {lost in } \Delta s}}{\Delta s \cdot N_{a b s}}
$$

High resolution loss maps are obtained, permitting the identification of the most critical regions of the ring. The performance of the LHC collimation system and the possible collimator-induced constraints are then analyzed for several commissioning scenarios. All the studies presented in the following sections refer to betatron halo simulations for the ideal machine.

\section{CLEANING PERFORMANCE}

Losses of particles overcoming the quench limit of the sc-magnets can imply a limitation in the beam intensity. The relation between the maximum number of particles circulating in the machine without causing quench $\left(\mathrm{N}_{\max }\right)$ and $\eta_{c}$ is:

$$
N_{\max }=\frac{\tau \cdot R_{q}}{\eta_{c}}
$$

Here $\tau$ is the beam life time and $\mathrm{R}_{q}$ is the quench threshold for continuous losses on the sc-magnets $\left(\mathrm{R}_{q}=7 \cdot 10^{8} \mathrm{p} /(\mathrm{m} \cdot \mathrm{s})\right.$ at $450 \mathrm{GeV}$ and $\mathrm{R}_{q}=7.8 \cdot 10^{6} \mathrm{p} /(\mathrm{m} \cdot \mathrm{s})$ at $7 \mathrm{TeV})$. From this formula the minimum local cleaning inefficiency $\eta_{q}$ required for running the machine at its nominal intensity $\left(\mathrm{I}_{\text {nom }}=3.23 \cdot 10^{14}\right.$ protons $)$ can be derived as function of $\tau$ :

- $450 \mathrm{GeV}: \eta_{q}=7.8 \cdot 10^{-4} \mathrm{~m}^{-1}$ for $\tau=0.1 \mathrm{~h}$.

- $7 \mathrm{TeV}: \eta_{q}=1.7 \cdot 10^{-5} \mathrm{~m}^{-1}$ for $\tau=0.2 \mathrm{~h}$.

The most critical region of the machine is the dispersion suppressor just downstream the betatron cleaning insertion. Particles impacting on the TCP can experience single diffractive scattering, loosing part of their energy and missing to intercept the down-stream TCSG and the TCLA. These off-momentum particles go straight forward towards the IR7 arc and are lost as soon as the dispersion increases. 


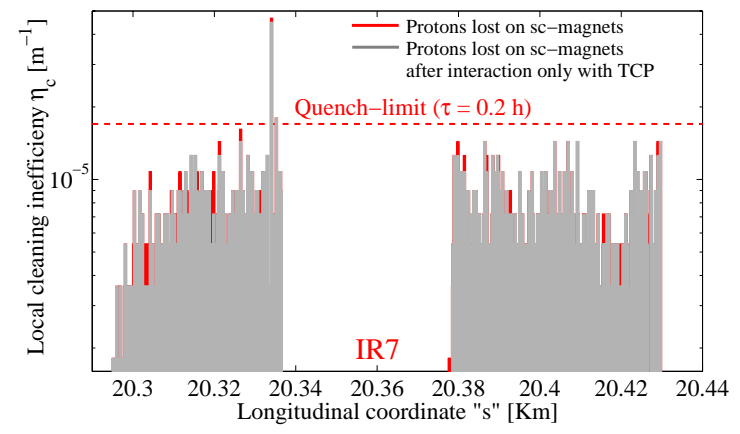

Figure 1: Proton loss map for B1 vertical halo at collision optics (zoom on IR7). About $90 \%$ the total losses on the sc-magnets of the IR7 dispersion suppressor (red bars) come from particles experiencing single diffractive scattering at the TCP(gray bars).

Fig. 1 shows the proton losses on the IR7 sc-magnets for the ideal machine at the nominal $7 \mathrm{TeV}$ collision optics (Beam1(B1), vertical halo). About $90 \%$ of these losses come from particles interacting only with the TCP. The cleaning performance of the collimation system, for this optics and for an assumed $\tau$ of $0.2 \mathrm{~h}$, allows to reach a maximum beam intensity $\left(\mathrm{I}_{\max }\right)$ of $\sim 46 \pm 6 \% \mathrm{I}_{n o m}$, where the statistical error is given. Machine imperfections, set-up errors and beam instabilities (closed orbit, beta beat, etc.) could further reduce the estimated $\mathrm{I}_{\max }$ of a factor 2 or more. The evaluation of $\mathrm{I}_{\max }$ depends on several parameters (diffusion length of the losses, $\tau, \mathbf{R}_{q}$ etc.) and the values reported in this paper refer to conservative hypotheses. At injection energy simulations predict that losses are below the quench limit.

\section{COLLISION AT $450 \mathrm{GEV}$}

For the early stages of the LHC commissioning the option of bringing the two low intensity beams $\left(<1 \% \mathrm{I}_{\text {nom }}\right)$ into collision at $450 \mathrm{GeV}$ is considered. The reduced number of circulating bunches (43) allows head-on collision (no crossing angle) without parasitic interactions. The beta function at the IP is the nominal injection $\beta^{*}: 11 \mathrm{~m}$ in IP1 (ATLAS) and IP5 (CMS), $10 \mathrm{~m}$ in IP2 (ALICE) and IP8 (LHC-b). A minimal collimation system is assumed. It consists of 8 TCP, 18 TCSG and 2 TCT in IR8 plus all the protectors in the dumping region. The collimators are at their nominal $450 \mathrm{GeV}$ setting (see Table 1) but with the TCT at $17 \sigma$. Simulations were performed for this optics and with this minimal setting of collimators for B1 and Beam2(B2), horizontal and vertical halo. The results show that this reduced system can insure the safe operation of the machine during the low intensity $450 \mathrm{GeV}$ run also in case of collision. A maximum $\eta_{c}$ of $\sim 1 \cdot 10^{-3}\left[\mathrm{~m}^{-1}\right]$ is predicted.

\section{COLLIMATION IN ENERGY RAMP}

After the commissioning of the machine at injection energy the acceleration ramp must be performed. The step from the $450 \mathrm{GeV}$ collimator setting to the $7 \mathrm{TeV}$ setting must be defined. At this stage the beam can have a reduced life time for a few seconds $(\tau=0.1 \mathrm{~h})$.

The tolerance budget $\mathrm{T}_{\text {coll }}$ of the collimation system is [3]:

$$
T_{\text {coll }}=n 2-n 1-0.4 \sigma
$$

here $\mathrm{n} 2$ and $\mathrm{n} 1$ are the TCSG and TCP half-gaps in $\sigma$ units and $0.4 \sigma$ is the minimum acceptable offset between $\mathrm{n} 2$ and $\mathrm{n} 1$. For the $450 \mathrm{GeV}$ setting $\mathrm{T}_{\text {coll }} \sim 0.6 \mathrm{~mm}$, while for the nominal top-energy setting $\mathrm{T}_{\text {coll }} \sim 0.12 \mathrm{~mm}$. Relaxed tolerances are desired during the energy ramp.

Different set-up options were studied for the complete phase 1 system. The optics and the available aperture of the machine do not change during the energy ramp and before the squeeze of the beam at the IP. Moreover the beam is adiabatically dumped during the acceleration.
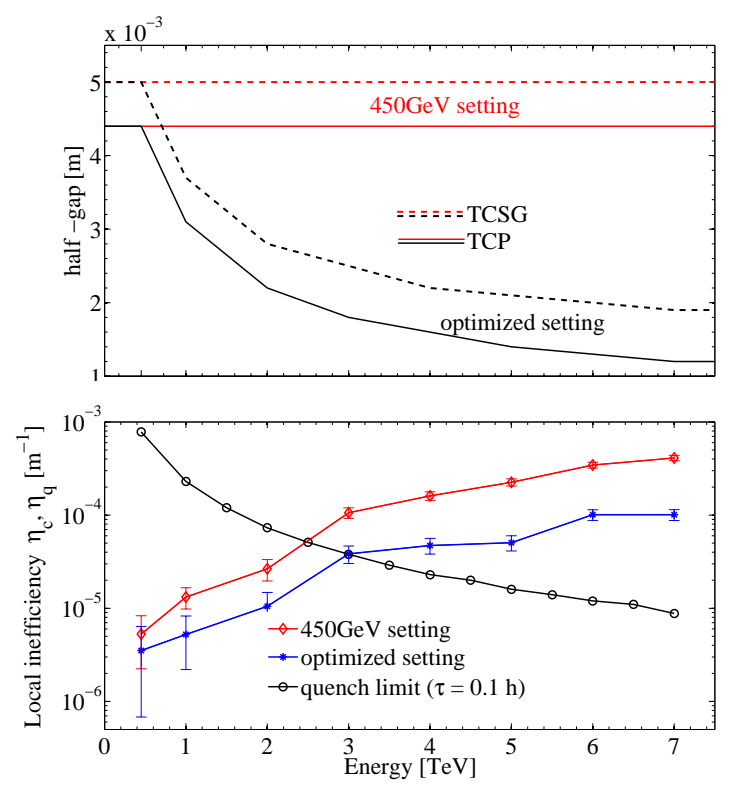

Figure 2: Top graph: TCP and TCSG half-gaps for the $450 \mathrm{GeV}$ and the optimized settings during the energy ramp. Bottom graph: comparison between $\eta_{c}$ and $\eta_{q}(\tau=0.1 \mathrm{~h})$ for the two settings at different energy. The error bars represent statistical errors.

One possible scenario is to close the collimators only after the end of the ramp before the change of the optics [4]. In this case $\mathrm{T}_{\text {coll }}$ at the end of the ramp is $\sim 0.9 \mathrm{~mm}$. Simulations performed for the ideal machine at several energies, with the collimators at the $450 \mathrm{GeV}$ setting, show (see Fig.2) the worsening of the local cleaning inefficiency with $\mathrm{E}$ (reduction of $\mathrm{I}_{\max }$ down to $\sim 2 \% \mathrm{I}_{\text {nom }}$ at $7 \mathrm{TeV}$ ). The results do not include losses of uncaptured particles at the start of the ramp. This setting could only be adopted for low intensity while for higher intensities the collimators should be closed before reaching the $7 \mathrm{TeV}$ plateau. An option is to move inwards the collimators driving the TCP down to $6 \sigma$ (optimized setting). The offset (in $\mathrm{mm}$ ) between the TCP and all the other collimators is kept unchanged. With this setting the inefficiency is much improved (see Fig.2 ) 
and $\mathrm{T}_{\text {coll }}$ is equal to $\sim 0.9 \mathrm{~mm}$ during the full ramp. The number of the single diffracted particles lost in the IR7 dispersion suppressor depends on the optics, on the beam energy and on the collimator settings.

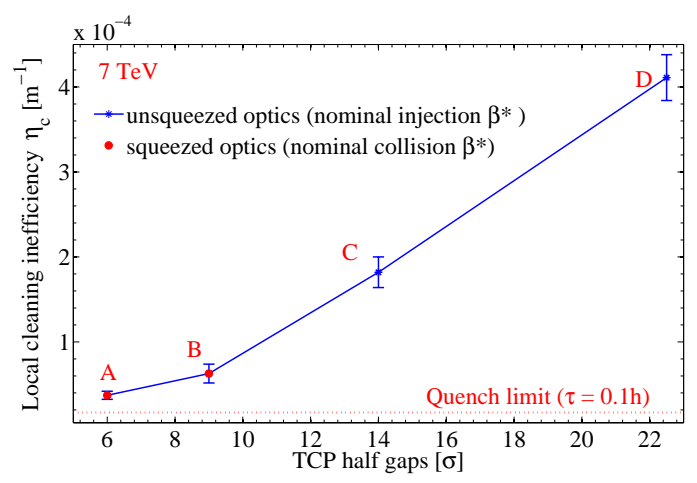

Figure 3: $\eta_{c}$ plotted as function of the TCP half-gaps for several settings of collimators at $7 \mathrm{TeV}$ and for squeezed (A,B) and unsqueezed (C,D) optics. The cleaning efficiency gets worse when the TCP are open. The error bars represent only statistical errors.

Fig. 3 shows the maximum value of $\eta_{c}$ (always in IR7) as function of the TCP half-gap for different optics and collimation settings:

- A: TCP/TCSG at $6 / 7 \sigma, \beta^{*}=0.55 \mathrm{~m}$.

- B: TCP/TCSG at 9/10 $\sigma, \beta^{*}=0.55 \mathrm{~m}$ (LHC upgrade studies).

- C: TCP/TCSG at $14 / 19 \sigma, \beta^{*}=11 \mathrm{~m}$.

- D: TCP/TCSG at $22.5 / 26.5 \sigma, \beta^{*}=11 \mathrm{~m}$.

\section{EARLY COLLISION OPTICS}

Before the squeeze of the beam down to the nominal $\beta^{*}$ of $0.55 \mathrm{~m}$ several intermediate $7 \mathrm{TeV}$ collision optics are foreseen. This section summarizes and completes studies on possible set-up sequences of the collimators for the $2 \mathrm{~m} \beta^{*}$ case (presented in more details in [5]).

In Fig.4 the TCP, TCSG, TCLA and TCSG@IR6 halfgaps and the $\mathrm{I}_{\max }$ are presented for different settings (B2 horizontal halo). For all these scenarios the TCT are kept at $17 \sigma$ and the offset between the IR6 collimators is the nominal one (see Table1). $\mathrm{I}_{\max }$ is evaluated considering also the energy deposition of the collimator induced showers on the sc-magnets (FLUKA simulations). When the betatron insertion is reduced to a one stage cleaning system (scenario 1 and scenarios 2) $\mathrm{I}_{\max }$ is $\leq 3 \% \mathrm{I}_{\text {nom }}$. This limitation is due to the showers of particles coming out from the IR6 collimators acting as secondary (see also scenarios $3 a$ and 3b) [5]. This energy load is problematic for $\mathrm{B} 2$ because the dumping region is downstream the betatron cleaning insertion. Losses in the IR7 dispersion suppressor become the limiting factor only when the IR6 collimators are in the shadow of the TCSG. For this case of B2 horizontal halo, $33 \% \mathrm{I}_{\text {nom }}(\tau=0.2 \mathrm{~h})$ can be reached (scenario3c), with relaxed tolerances, keeping the secondary collimators

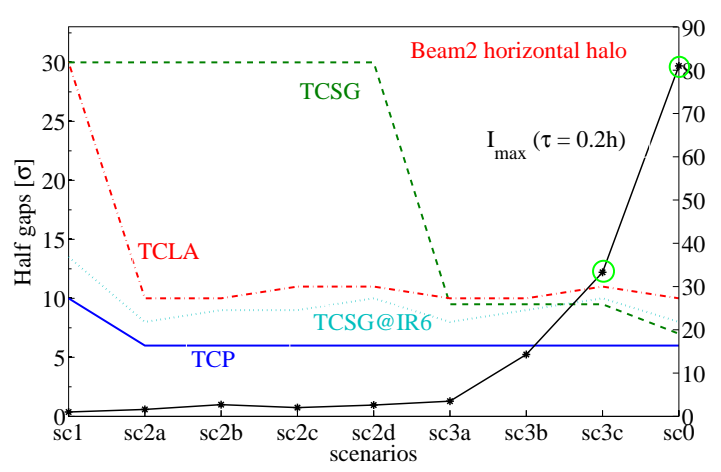

Figure 4: The beam intensity $I_{\max }$ is limited by the possible quench of the IR6 sc-magnets for all the commissioning scenarios except sc $3 \mathrm{c}$ and $\mathrm{sc} 0$ (circled points). In this case losses in IR7 play the dominant role.

at $9.5 \sigma$. Closing the TCSG at the nominal $7 \mathrm{TeV}$ setting $81 \% \mathrm{I}_{\text {nom }}$ can be reached.

These studies highlight the crucial role played by the TCSG in the protection of the machine and of other more critical collimators.

\section{CONCLUSIONS}

Different stages of the LHC collimation system commissioning have been considered. The number of collimators and their settings were varied, reflecting the options of commissioning with (1) a reduced set of collimators or (2) with all collimators but relaxed settings. It has been shown that a sub-set of collimators (34 out of 88) is sufficient to clean the $450 \mathrm{GeV}$ LHC beams with 43 bunches. Commissioning scenarios, that use many collimators but somewhat relaxed collimator gaps, have been proven to provide maximum tolerances to the machine operation (beta beat, orbit, collimator set-up tolerances) for the energy ramp and the $7 \mathrm{TeV}$ commissioning. Controls software is being implemented to provide the necessary flexibility and precision to drive the collimation system through the stages defined in this paper. These commissioning studies will allow reducing the time required for setting the LHC collimation during the first years of the LHC operation. Further ongoing studies will focus on (1) simulating imperfect cleaning and (2) on the requirements from momentum cleaning.

\section{REFERENCES}

[1] The LHC Design Report, Vol.1, Chapter 18. CERN-2004003, pp. 467-498.

[2] G.Robert-Demolaize et al., "A new version of SixTrack with collimation and aperture interface", PAC 2005, CERN-AB2005-033.

[3] R.Assmann, "Beam commissioning of the collimation system", ChamonixXV, CERN-AB-2006-014, pp.101-108.

[4] R.Assmann, "Collimators and Beam Absorbers for Cleaning and Machine Protection", ChamonixXIV, CERN-AB-2005014, pp. 261-267.

[5] R.Bailey, "LHC collimation efficiency during commissioning”, EPAC06, CERN-LHC-PROJECT-Report-920. 